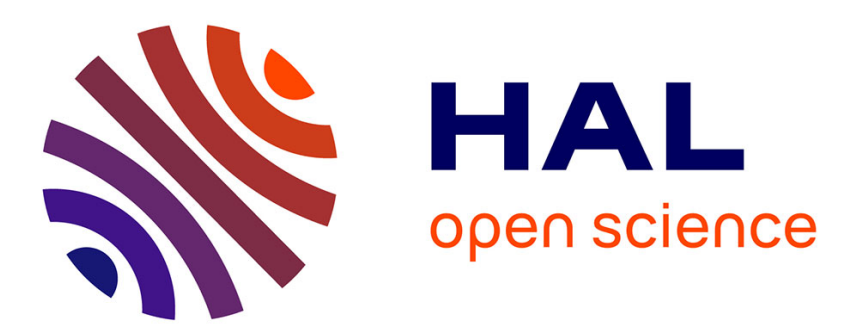

\title{
Estimating Biophysical Parameters from BOLD Signals through Evolutionary-Based Optimization
}

\author{
Pablo Mesejo, Sandrine Saillet, Olivier David, Christian Bénar, Jan M. \\ Warnking, Florence Forbes
}

\section{To cite this version:}

Pablo Mesejo, Sandrine Saillet, Olivier David, Christian Bénar, Jan M. Warnking, et al.. Estimating Biophysical Parameters from BOLD Signals through Evolutionary-Based Optimization. 18th International Conference on Medical Image Computing and Computer Assisted Intervention (MICCAI'15), Oct 2015, Munich, Germany. pp.528-535, 10.1007/978-3-319-24571-3_63 . hal-01221126

\section{HAL Id: hal-01221126 \\ https://hal.inria.fr/hal-01221126}

Submitted on 27 Oct 2015

HAL is a multi-disciplinary open access archive for the deposit and dissemination of scientific research documents, whether they are published or not. The documents may come from teaching and research institutions in France or abroad, or from public or private research centers.
L'archive ouverte pluridisciplinaire HAL, est destinée au dépôt et à la diffusion de documents scientifiques de niveau recherche, publiés ou non, émanant des établissements d'enseignement et de recherche français ou étrangers, des laboratoires publics ou privés. 


\title{
Estimating Biophysical Parameters from BOLD Signals through Evolutionary-based Optimization
}

\author{
Pablo Mesejo $^{1}$, Sandrine Saillet ${ }^{2,5}$, Olivier David ${ }^{2,5}$, Christian Bénar ${ }^{3,4}$, Jan M. \\ Warnking $^{2,5}$, and Florence Forbes ${ }^{1}$ \\ 1 INRIA, Univ. Grenoble Alpes, LJK, F-38000, Grenoble, France \\ pablo.mesejo-santiagoeinria.fr \\ 2 INSERM, U836, F-38000, Grenoble, France \\ 3 INSERM, UMR1106, Marseille, France \\ 4 Aix-Marseille Université, Institut de Neurosciences des Systèmes, Marseille, France \\ ${ }^{5}$ Univ. Grenoble Alpes, GIN, F-38000, Grenoble, France
}

\begin{abstract}
Physiological and biophysical models have been proposed to link neural activity to the Blood Oxygen Level-Dependent (BOLD) signal in functional MRI (fMRI). They rely on a set of parameter values that cannot always be extracted from the literature. Their estimation is challenging because there are more than 10 potentially interesting parameters involved in non-linear equations and whose interactions may result in identifiability issues. However, the availability of statistical prior knowledge on these parameters can greatly simplify the estimation task. In this work we focus on the extended Balloon model and propose the estimation of 15 parameters using an Evolutionary Computation (EC) global search method. To combine both the ability to escape local optima and to incorporate prior knowledge, we derive the EC objective function from Bayesian modeling. This novel method provides promising results on a challenging real fMRI data set involving rats with epileptic activity and compares favorably with the conventional Expectation Maximization Gauss-Newton approach.
\end{abstract}

Keywords: functional MRI, BOLD signal, Biophysical parameters, Evolutionary Computation, Differential Evolution, Expectation Maximization.

\section{Introduction}

In the past decade, physiological models have been proposed to describe the processes that link the neural and the hemodynamic activity in the brain. Different variations of the widely used "Balloon model" [2] have been introduced that provide a complete description of the physiological processes underlying hemodynamic activity, from neural activation to the Blood-Oxygen-Level-Dependent (BOLD) effect measurement [1]10]. These models all depend on physiological parameters for which different competing values have been proposed in the literature, e.g. [10[11]. Most approaches that focus on such models currently use one of these empirical sets of values with no real justification, e.g. [8]14], although it has been shown in [8], and to a lesser extent in [14], that the selection of these parameters had a more critical impact than the choice of the Balloon model variant itself, via their influence on the system dynamics. Estimating these physiological parameters from observed data may therefore be of interest in a number of fMRI 
studies. A general method for estimating parameters involved in a dynamic system has been proposed [9] based on a Bayesian approach which allows the incorporation of prior knowledge. Such a priori knowledge is typically summarized by a Gaussian distribution for each physiological parameter and provides a generally accepted consensus avoiding the commitment to arbitrarily fixed values. The method in [9] has then been widely used as the method of reference to estimate the hemodynamic response in dynamical causal modelling (DCM). It is based on an Expectation-Maximization Gauss-Newton search (EM/GN) which requires the linearization of the original system and approximation such as the Laplace approximation for tractability. The EM algorithm is also more generally known to be sensitive to initialization and prone to get stuck in local optima. Alternative approaches include sampling, e.g. Monte Carlo Markov Chain (MCMC), or other stochastic techniques, e.g. Metaheuristics (MHs). Sampling techniques offer a number of attractive features such as robust and reliable performance and ability to escape local optima. MHs are in addition general purpose procedures that do not even require the availability of the objective function in analytic form. In the DCM context, the need for the Laplace approximation is relaxed by [3] which uses a MCMC implementation of the Bayesian inversion scheme of [9] and shows that the Laplace approximation actually yields sensible inferences under a large set of conditions. However, MCMC needs thousands of iterations to converge, constraints are not easy to introduce and it does not provide mechanisms to control the trade-off exploration-exploitation. Also, [3] focuses on DCM and neuronal parameter estimation while nothing is reported on the impact on the non-neuronal physiological parameters. In contrast, in [16] the authors consider the Balloon model in a non-Bayesian setting using standard MHs with an objective, or so-called fitness function which does not include prior information. Without such valuable prior knowledge, it is quite challenging to put all parameters into the proposed optimization scheme due to potential identifiability issues. It results that the approach in [16] is limited to the estimation of three of the physiological parameters out of the 15 considered in this paper.

In this work, our goal is to combine both the benefits from a Bayesian approach which allows incorporation of prior knowledge and from MHs which are generalpurpose global optimization techniques able to avoid local optima. Following the Bayesian inversion scheme of [9], we derive a fitness function that is directly comparable to EM/GN search DCM standard. It follows an estimation procedure able to estimate all physiological parameters of interest while being less likely to get trapped in local minima. This novel method is assessed on a challenging real EEG/fMRI data set involving rats with epileptic activity. A qualitative comparison with the EM/GN approach shows the ability of our method to provide more physiologically sensible parameter values.

\section{The extended Balloon model}

The Balloon model was first proposed in [2] to link neuronal and vascular processes by considering the venous vascular compartment as a balloon that inflates under the effect of blood flow variations. More specifically, the model describes how, after some stimulation, the local blood flow $f_{\text {in }}(t)$ increases and leads to the subsequent augmentation of the local deoxygenated blood volume $v(t)$. The incoming blood is strongly oxygenated, 
and since the relative blood flow increase exceeds the increase in oxygen consumption, local deoxyhemoglobin concentration $q(t)$ decreases and induces a BOLD signal increase. The Balloon model was subsequently extended [10] to include the effect of the neuronal activity on the variation of some auto-regulated flow inducing signal $s(t)$ so as to eventually link neuronal to hemodynamic activity. Variable $n_{e}(t)$ represents the activity of the excitatory neuron population and $n_{i}(t)$ the inhibitory neuron population [12]. The experimentally controlled input function (stimulus) is represented by $u(t)$. In the following, the explicit time dependence ' $(t)$ ' of the state variables will be omitted for compactness. The global physiological model corresponds then to a non-linear system with six state variables $\boldsymbol{x}=\left\{n_{e}, n_{i}, s, f_{i n}, v, q\right\}$ related to the excitatory and inhibitory neuronal activity, normalized flow inducing signal, local blood flow, local deoxygenated blood volume, and deoxyhemoglobin concentration. Their interactions over time are described by the following non-linear differential equations:

$$
\begin{gathered}
\frac{d n_{e}}{d t}=-E n_{e}-\exp \left(A+B u^{s e}+\boldsymbol{D}^{T}\left(\begin{array}{c}
n_{e} \\
s \\
f_{i n}-1
\end{array}\right)\right) n_{i}+C u^{s e} \\
\frac{d n_{i}}{d t}=n_{e}-2 E n_{i}, \quad \frac{d s}{d t}=n_{e}-s d s-\operatorname{ar}\left(f_{i n}-1\right), \quad \frac{d \ln \left(f_{i n}\right)}{d t}=\frac{s}{f_{\text {in }}} \\
\frac{d \ln (v)}{d t}=\frac{1}{t t} \frac{f_{\text {in }}-v^{\frac{1}{\alpha}}}{v}, \quad \frac{d \ln (q)}{d t}=\frac{1}{t t}\left(\frac{1-\left(1-E_{0}\right)^{\frac{1}{f_{i n}}}}{E_{0}} \frac{f_{\text {in }}}{q}-v^{\frac{1}{\alpha}-1}\right)
\end{gathered}
$$

From these state variables, the observed BOLD signal $y$ is derived using an observation equation that includes intra- and extravascular BOLD signal components [1]:

$$
y=V_{0}\left[k_{1}(1-q)+k_{2}\left(1-\frac{q}{v}\right)+k_{3}(1-v)\right]
$$

where $k_{1}, k_{2}, k_{3}$ are physiology- and scanner-dependent constants $k_{1}=4.3 \theta_{0} E_{0} T E$, $k_{2}=\varepsilon r_{0} E_{0} T E$ and $k_{3}=1-\varepsilon$.

The value $\theta_{0}$ is the frequency offset at the outer surface of the magnetized vessel for fully deoxygenated blood, it is equal to $40.3 \mathrm{~Hz} \cdot b_{0} / 1.5 \mathrm{~T}$, where $b_{0}$ is the magnetic field strength. $T E$ is the echo time and $r_{0}$ is the slope of the relation between the intravascular relaxation rate and oxygen saturation, which is set to $300 \mathrm{~Hz}$ [13]. $E_{0}$ is the oxygen extraction fraction at rest and is considered as a free parameter as well as $\varepsilon$, the ratio of intra to extravascular signal.

The remaining parameters are the following: $A, B, C, \boldsymbol{D}$ and $E$ are parameters as in non-linear Dynamic Causal Models [15], $\boldsymbol{D}=\left(D_{1}, D_{2}, D_{3}\right)^{T}$ being the new component in the non-linear state equation above. Parameter se is the spike exponent introduced for the present dataset to control the scaling of the synaptic activity with respect to the spike amplitude derived from local field potentials (LFPs), $s d$ is the vasodilatory signal decay, $a r$ is the rate constant for autoregulatory feedback by blood flow, and $t t$ represents the transit time of blood from the arteriolar to the venous compartment. The Grubb's vessel stiffness exponent corresponds to $\alpha$, while $V_{0}$ is the resting venous cerebral blood volume fraction. The whole model depends on 15 different scalar parameters to optimize $\boldsymbol{\theta}=\left\{A, B, C, \boldsymbol{D}, E, s e, s d, a r, t t, \alpha, E_{0}, V_{0}, \varepsilon\right\}$. 


\section{Bayesian estimation of dynamical systems}

MHs require the definition of a fitness function to measure the goodness of the parameters found. We use the Bayesian inversion scheme of [9] to derive an appropriate fitness function. In the Balloon model, the first part describes the transitional dynamics of the state vector $\boldsymbol{x}=\left\{n_{e}, n_{i}, s, f_{i n}, v, q\right\}$. The system is defined as $\frac{d \boldsymbol{x}}{d t}=f(\boldsymbol{x}, \boldsymbol{u}, \boldsymbol{\psi})$, with $\boldsymbol{\psi}=\left\{A, B, C, \boldsymbol{D}, E, s e, s d, a r, t t, \alpha, E_{0}\right\}$. The second part of the model is the observational equation for the BOLD signal $\boldsymbol{y}$ which is assumed to be observed with some additive Gaussian noise (in this context of BOLD data sampled at discrete time points, we represent both data and state variables as vectors of discrete samples), $\boldsymbol{y}=g(\boldsymbol{x}, \boldsymbol{\phi})+\boldsymbol{\eta}$, with $\phi=\left\{V_{0}, E_{0}, \varepsilon\right\}$ and $\boldsymbol{\eta}$ is a random error vector distributed according to the Gaussian distribution $\mathcal{N}\left(0, \sigma_{\eta}^{2} \mathbf{I}\right)$ assuming unstructured noise. Under additional distributional assumptions about the model parameters $\boldsymbol{\theta}=\{\boldsymbol{\psi}, \boldsymbol{\phi}\}$ and noise variance $\sigma_{\eta}^{2}$, we can apply Bayesian inference. In [9], Gaussian priors are chosen for all parameters. As explained in [14] for $\varepsilon$, it is more natural to use log-normal priors for parameters that are positive. A simple way to account for positivity while remaining in a Gaussian setting is to change the model parameterization. We consider equivalently $\tilde{\boldsymbol{\theta}}=$ $\left\{\tilde{A}, \tilde{B}, \tilde{C}, \tilde{\boldsymbol{D}}, \tilde{E}, \tilde{s e}, \tilde{s d}, \tilde{a r}, \tilde{t t}, \tilde{\alpha}, \tilde{E}_{0}, \tilde{V_{0}}, \tilde{\varepsilon}\right\}$, where $\{\tilde{A}, \tilde{B}, \tilde{C}, \tilde{\boldsymbol{D}}\}=\{A, B, C, \boldsymbol{D}\}$ remain unchanged while the other parameters take the form $\tilde{\theta}=\log \left(\theta / \mu_{\theta}\right)$ where the specific $\mu_{\theta}$ values may depend on the experiment (see section 5). An exception is $E_{0}$ for which we set $E_{0}=\arctan \left(\tilde{E}_{0}+\tan \left(\pi\left(\mu_{E_{0}}-0.5\right)\right)\right) / \pi+0.5$ in order to ensure $E_{0} \in$ $[0,1]$. Gaussian priors can then be assumed for $\tilde{\boldsymbol{\theta}}$ and the state and observational equations above lead to, $\boldsymbol{y}=h(\tilde{\boldsymbol{\theta}}, \boldsymbol{u})+\boldsymbol{\eta}$, with $\boldsymbol{\eta} \sim \mathcal{N}\left(0, \sigma_{\eta}^{2} \mathbf{I}\right), \tilde{\boldsymbol{\theta}} \sim \mathcal{N}\left(\overline{\boldsymbol{\theta}}, \boldsymbol{\Sigma}_{\tilde{\theta}}\right)$ and $\sigma_{\eta}^{2} \sim$ $p\left(\sigma_{\eta}^{2}\right)$. As another difference with [9]14], we use a semi-conjugate prior for the unknown parameters $\left(\tilde{\boldsymbol{\theta}}, \sigma_{\eta}^{2}\right)$ in which $\tilde{\boldsymbol{\theta}} \sim \mathcal{N}\left(\overline{\boldsymbol{\theta}}, \boldsymbol{\Sigma}_{\tilde{\theta}}\right)$ independently of $\sigma_{\eta}^{2}$ and a noninformative prior is used for $\sigma_{\eta}^{2}$, i.e. $p\left(\sigma_{\eta}^{2}\right) \propto\left(\sigma_{\eta}^{2}\right)^{-1}$. Bayesian inference is then based on the posterior distribution $p\left(\tilde{\boldsymbol{\theta}}, \sigma_{\eta}^{2} \mid \boldsymbol{y}\right) \propto p\left(\boldsymbol{y} \mid \tilde{\boldsymbol{\theta}}, \sigma_{\eta}^{2}\right) p(\tilde{\boldsymbol{\theta}}) p\left(\sigma_{\eta}^{2}\right)$ whose mode provides the maximum a posteriori (MAP) estimate:

$$
\begin{aligned}
\left(\tilde{\boldsymbol{\theta}}, \sigma_{\eta}^{2}\right)_{M A P} & =\arg \max _{\tilde{\boldsymbol{\theta}}, \sigma_{\eta}^{2}}\left\{\log p\left(\boldsymbol{y} \mid \tilde{\boldsymbol{\theta}}, \sigma_{\eta}^{2}\right)+\log p(\tilde{\boldsymbol{\theta}})+\log p\left(\sigma_{\eta}^{2}\right)\right\} \\
& =\arg \min _{\tilde{\boldsymbol{\theta}}, \sigma_{\eta}^{2}}\left\{(N+2) \log \sigma_{\eta}^{2}+\frac{\|\boldsymbol{y}-h(\tilde{\boldsymbol{\theta}}, \boldsymbol{u})\|^{2}}{\sigma_{\eta}^{2}}+(\tilde{\boldsymbol{\theta}}-\overline{\boldsymbol{\theta}})^{T} \boldsymbol{\Sigma}_{\tilde{\theta}}^{-1}(\tilde{\boldsymbol{\theta}}-\overline{\boldsymbol{\theta}})\right\}
\end{aligned}
$$

where $N$ is the $\boldsymbol{y}$ signal length. Setting to zero the gradient with respect to $\sigma_{\eta}^{2}$ yields

$$
\begin{aligned}
\left(\sigma_{\eta}^{2}\right)_{M A P} & =\frac{\left\|\boldsymbol{y}-h\left(\tilde{\boldsymbol{\theta}}_{M A P}, \boldsymbol{u}\right)\right\|^{2}}{N+2} . \text { Plugin in }\left(\sigma_{\eta}^{2}\right)_{M A P} \text { into expression } 3 \text { leads to } \\
\tilde{\boldsymbol{\theta}}_{M A P} & =\arg \min _{\tilde{\boldsymbol{\theta}}}\left\{(N+2) \log \|\boldsymbol{y}-h(\tilde{\boldsymbol{\theta}}, \boldsymbol{u})\|^{2}+(\tilde{\boldsymbol{\theta}}-\overline{\boldsymbol{\theta}})^{T} \boldsymbol{\Sigma}_{\tilde{\theta}}^{-1}(\tilde{\boldsymbol{\theta}}-\overline{\boldsymbol{\theta}})\right\} .
\end{aligned}
$$

The left-hand side above corresponds to our fitness function to be used in the EC framework described in the next section. In contrast to the conventional Laplace approximation and EM estimation algorithm, EC does not require the linearization or approximation of $h(\tilde{\boldsymbol{\theta}}, \boldsymbol{u})$. It does not require an analytic form of the likelihood and $h(\tilde{\boldsymbol{\theta}}, \boldsymbol{u})$ can typically be used as a numerical function. Another advantage of EC is its flexibility in particular as regards hard constraints often imposed for stability of the differential equations $(1)$. The hyperparameters $\overline{\boldsymbol{\theta}}$ and $\boldsymbol{\Sigma}_{\theta}$ are specified in section 5 . 


\section{Evolutionary Computation}

Evolutionary Computation (EC) methods are population-based MH algorithms [7]. They include several computational models that reproduce natural evolution processes to reach a target which is generally represented as a fitness function to optimize. In practice, they implement an iterative process (artificial evolution) in which solutions improve over generations until they converge to an optimum, starting from an initial pool of randomly generated solutions. EC procedures are based on achieving a trade-off between intensification (exploitation of the best solutions, usually through selection operators and replacement strategies) and diversification (exploration of the search space thanks to crossover and mutation operators).

In this work, we choose to use Differential Evolution (DE) [5], which has recently been shown to be one of the most successful EC methods for global continuous optimization. DE perturbs individuals in the current generation by the scaled differences of other randomly selected and distinct individuals. In DE, each individual acts as a parent vector, and for each of them a new solution, called donor vector, is created. In the basic version of DE, the donor vector for the $i^{t h}$ parent $\left(\tilde{\boldsymbol{\theta}}_{i}\right)$ is generated by combining three random and distinct elements $\tilde{\boldsymbol{\theta}}_{r 1}, \tilde{\boldsymbol{\theta}}_{r 2}$ and $\tilde{\boldsymbol{\theta}}_{r 3}$. The donor vector $\boldsymbol{V}_{i}$ is computed as $\boldsymbol{V}_{i}=\tilde{\boldsymbol{\theta}}_{r 1}+F \cdot\left(\tilde{\boldsymbol{\theta}}_{r 2}-\tilde{\boldsymbol{\theta}}_{r 3}\right)$, where $F$ (scale factor) is a parameter that strongly influences DE's performance and typically lies in the interval $[0.4,1]$. The original method described above is called DE/rand/1, which means that the first element of the donor vector equation $\tilde{\boldsymbol{\theta}}_{r 1}$ is randomly chosen and only one difference vector (in this case $\tilde{\boldsymbol{\theta}}_{r 2}-\tilde{\boldsymbol{\theta}}_{r 3}$ ) is added. After mutation, every parent-donor pair generates a child (called trial vector) by means of a crossover operation. The crossover is applied with a certain probability, defined by a parameter $C r$ (crossover rate) that, like $F$, is one of the control parameters of DE. Then, the trial vector is evaluated and its fitness is compared to the parent's. The best, in terms of fitness, survives and will be part of the next generation.

\section{Experimental results}

The BOLD data used in the experiments was recorded with the goal of testing biophysical models in the context of epileptic activity in rats. An intracortical silica capillary was surgically implanted in the right primary somatosensory cortex of male Wistar rats $(\sim 400 \mathrm{~g})$ and subdural carbon EEG electrodes were placed close to the injection site and over the cerebellum. Epileptic activity was elicited using bicuculline methochloride ( $2.5 \mathrm{mM}, 1 \mu \mathrm{l} / 5 \mathrm{~min}$ ) injected intra-cortically during the MRI session. Simultaneous EEG and BOLD-fMRI data were acquired under $<2 \%$ isoflurane anesthesia.

The EEG/fMRI data were acquired on a $4.7 \mathrm{~T}$ Advance III Bruker Biospec.In each scan, 300 volumes of five slices $\left(0.25 \times 0.25 \times 0.8 \mathrm{~mm}^{3}\right.$ voxel size $)$ were acquired using single-shot GE EPI with TE/TR of 20/600 ms. A total of 3-12 scans were performed for each of 12 rats ( $27 \mathrm{~min}$ of EEG/fMRI data per animal on average). The data from 3 animals were unexploitable and thus excluded from the analysis. Epileptic discharges (EDs) were automatically identified from the EEG data and ED amplitudes and onsets were recorded. For each rat, a single average fMRI signal concatenating all scans was extracted from the largest cluster of significantly active voxels identified in a linear 
analysis with a FIR hemodynamic response model. The fMRI signal size $N$ ranged from 894 to 3576 with a median value of 2684 . The EDs were entered in the biophysical model via the input function $\boldsymbol{u}$ as a series of short ( $8 \mathrm{~ms}$ ) events.

To palliate the absence of ground truth (GT) in real data, a synthetic dataset is created to study the methods behavior under controlled conditions. Rat 5, whose physiological conditions are amongst the most stable ones, is selected as a reference to create this dataset, and the parameter estimates found by EM/GN are defined as the GT. BOLD signals are generated from either a full set of measured spikes or a subset $(25 \%)$ to simulate more sparse events, adding $A R(1)$ noise with three target SNRs $(0.10,0.46$ and 2.15). The average distance to GT using $25 \%$ subsampling and the full set of spikes are 0.56 and 2.8 in $\mathrm{EM} / \mathrm{GN}$, and $0.28 \pm 0.09$ and $0.15 \pm 0.01$ in $\mathrm{DE}$, respectively.

Then, for each rat, physiological parameters $\theta$ are estimated using DE with fitness function (4) and transforming back the resulting $\tilde{\boldsymbol{\theta}}$ into $\boldsymbol{\theta}$. Each of the positive parameters in $\boldsymbol{\theta}$ is specified using $\tilde{\theta}=\log \left(\theta / \mu_{\theta}\right)$ with $\mu_{\theta}$ defined respectively by $\left\{\mu_{E}, \mu_{s e}, \ldots, \mu_{\varepsilon}\right\}=\{1,1,0.64,0.41,0.98,0.32,0.55,0.4,1\}$ [6]. The prior means are then set to $\overline{\boldsymbol{\theta}}=\mathbf{0}$ and the prior covariance $\boldsymbol{\Sigma}_{\tilde{\theta}}$ is a diagonal matrix containing the prior variances set to $\{0.25,0.25,54.6,0.05,0.05,0.05,0.05,0.14,0.14,0.05,0.05,0.007$, $0.007,0.05,0.14\}$ for each parameter in $\tilde{\boldsymbol{\theta}}$. The DE parameters used are among the most common ones in the state of the art [5]: $F=0.85, C r=1$, with a DE/local-to-best/1 strategy that attempts a balance between robustness and fast convergence, and a population size of 150 . The EM/GN algorithm [9] starts from physiologically reasonable parameter values (the prior means), which facilitates its convergence to a good solution. Since DE is a stochastic approach several runs need to be executed to evaluate its average performance. In this study, the number of runs per rat and the number of iterations per run are empirically set to 15 and 300, respectively. DE shows a very stable behavior with a standard deviation of the mean fitness values between $0.001 \%$ and $0.042 \%$.

The $\boldsymbol{\theta}$ parameter estimations from DE and EM/GN are shown in Table 1 . The experimental conditions for all animals were controlled as closely as possible. It is therefore expected that the physiologically meaningful parameters show limited variability across animals. However, the parameters related to the scaling of the stimulus and the neuronal signals (notably, $A, B, C, D, E$ and $s e$ ) may vary significantly, since the amplitudes of the elicited EEG responses varied between sessions. Estimates for 4 of the parameters that are expected to be among the most stable ones are shown in Figure 1. The ratio between intra- and extravascular signals, $\varepsilon$, depends on field strength, blood $\mathrm{T}_{2}$, and vascular geometry, but little on physiology. The values estimated by DE are markedly more stable between animals than the values obtained with EM/GN. Quantitatively, both methods yield plausible results. Blood transit times from arterioles to the deoxygenated vascular compartment estimated with DE are generally longer, and closer to the expected value, than those obtained with EM/GN, which are rather too short. For comparison, mean transit times across the entire vascular tree in a cortical voxel observed using DSC MRI in anesthesized rats are on the order of $1.6 \mathrm{~s}$ [4]. Inversely, resting venous blood volumes estimated with $\mathrm{DE}$ are lower than those obtained with EM/GN. The prior estimate used here for this parameter, $4 \%$, corresponds to the total cortical resting blood volume in isoflurane anesthesized male Wistar rats [4]. In hindsight, the value that should actually be considered in the model is however only the 


\begin{tabular}{c|c|c|c|c|c|c|c|c|c|c|c|c|c|c|c|} 
& $A$ & $B$ & $C$ & $D_{1}$ & $D_{2}$ & $D_{3}$ & $E$ & $s e$ & $s d$ & $a r$ & $t t$ & $\alpha$ & $V_{0}$ & $E_{0}$ & $\varepsilon$ \\
\hline RAT1 DE & 0.56 & 0.02 & 0.10 & 0.01 & 0.02 & 0.05 & 0.84 & 1.44 & 0.89 & 0.53 & 0.83 & 0.32 & 0.38 & 0.55 & 0.90 \\
EM/GN & 0.14 & -0.01 & 0.02 & 0.01 & 0.01 & 0.01 & 0.86 & 1.11 & 0.61 & 0.44 & 0.65 & 0.31 & 0.53 & 0.55 & 1.77 \\
\hline RAT2 DE & 0.48 & 0.03 & 3.00 & -0.14 & -0.10 & 0.46 & 0.73 & 0.40 & 2.02 & 0.25 & 0.91 & 0.35 & 0.20 & 0.53 & 0.35 \\
EM/GN & 0.03 & -0.00 & 0.02 & 0.01 & 0.01 & 0.01 & 0.96 & 0.60 & 0.73 & 0.36 & 0.44 & 0.30 & 0.58 & 0.54 & 2.13 \\
\hline RAT3 DE & -0.66 & -0.01 & 3.00 & -0.14 & -0.09 & 0.58 & 0.73 & 0.96 & 1.00 & 0.26 & 0.85 & 0.33 & 0.15 & 0.52 & 0.42 \\
EM/GN & 1.35 & 0.02 & 0.26 & 0.07 & -0.01 & 0.18 & 0.72 & 0.83 & 1.12 & 0.59 & 0.65 & 0.32 & 0.34 & 0.54 & 0.94 \\
\hline RAT4 DE & -0.14 & 0.01 & 2.18 & 0.31 & -0.43 & 0.05 & 0.40 & 0.50 & 1.95 & 0.36 & 0.72 & 0.33 & 0.15 & 0.54 & 0.41 \\
EM/GN & -0.14 & 0.01 & 2.10 & 0.33 & -0.42 & 0.07 & 0.41 & 0.50 & 1.86 & 0.35 & 0.71 & 0.33 & 0.16 & 0.54 & 0.41 \\
\hline RAT5 DE & 1.11 & 0.03 & 1.60 & -0.08 & -0.11 & 0.17 & 0.57 & 0.82 & 2.05 & 0.46 & 0.67 & 0.35 & 0.23 & 0.54 & 0.36 \\
EM/GN & 1.07 & 0.03 & 1.21 & -0.09 & -0.12 & 0.19 & 0.56 & 0.78 & 2.07 & 0.46 & 0.65 & 0.34 & 0.26 & 0.55 & 0.39 \\
\hline RAT6 DE & -0.85 & 0.01 & 3.00 & 0.23 & 0.24 & 0.18 & 0.44 & 0.33 & 1.15 & 0.25 & 0.97 & 0.33 & 0.22 & 0.52 & 0.49 \\
EM/GN & 0.21 & -0.01 & 0.03 & 0.03 & 0.04 & 0.03 & 0.71 & 1.13 & 1.04 & 0.39 & 0.38 & 0.30 & 0.62 & 0.55 & 2.38 \\
\hline RAT7 DE & 1.75 & -0.03 & 0.09 & -0.00 & 0.00 & 0.01 & 0.65 & 1.23 & 1.30 & 0.47 & 0.65 & 0.32 & 0.40 & 0.55 & 1.16 \\
EM/GN & 1.70 & -0.03 & 0.07 & -0.00 & 0.00 & 0.01 & 0.65 & 1.23 & 1.25 & 0.47 & 0.64 & 0.31 & 0.42 & 0.55 & 1.30 \\
\hline RAT8 DE & 0.10 & 0.07 & 2.99 & -0.43 & -0.47 & -0.02 & 0.39 & 0.19 & 1.92 & 0.14 & 0.60 & 0.31 & 0.22 & 0.53 & 0.49 \\
EM/GN & -0.67 & 0.08 & 0.30 & -0.08 & -0.10 & 0.11 & 0.50 & 0.14 & 1.40 & 0.57 & 0.51 & 0.31 & 0.32 & 0.54 & 1.14 \\
\hline RAT9 DE & 0.74 & 0.03 & 0.55 & 0.02 & -0.01 & 0.06 & 0.45 & 0.68 & 2.74 & 0.38 & 0.58 & 0.32 & 0.36 & 0.55 & 0.60 \\
EM/GN & 0.74 & 0.03 & 0.52 & 0.01 & -0.01 & 0.07 & 0.45 & 0.68 & 2.71 & 0.38 & 0.57 & 0.32 & 0.37 & 0.55 & 0.62 \\
\hline
\end{tabular}

Table 1. EM estimates and DE medians for each of the 9 rats and each parameter in $\boldsymbol{\theta}$.
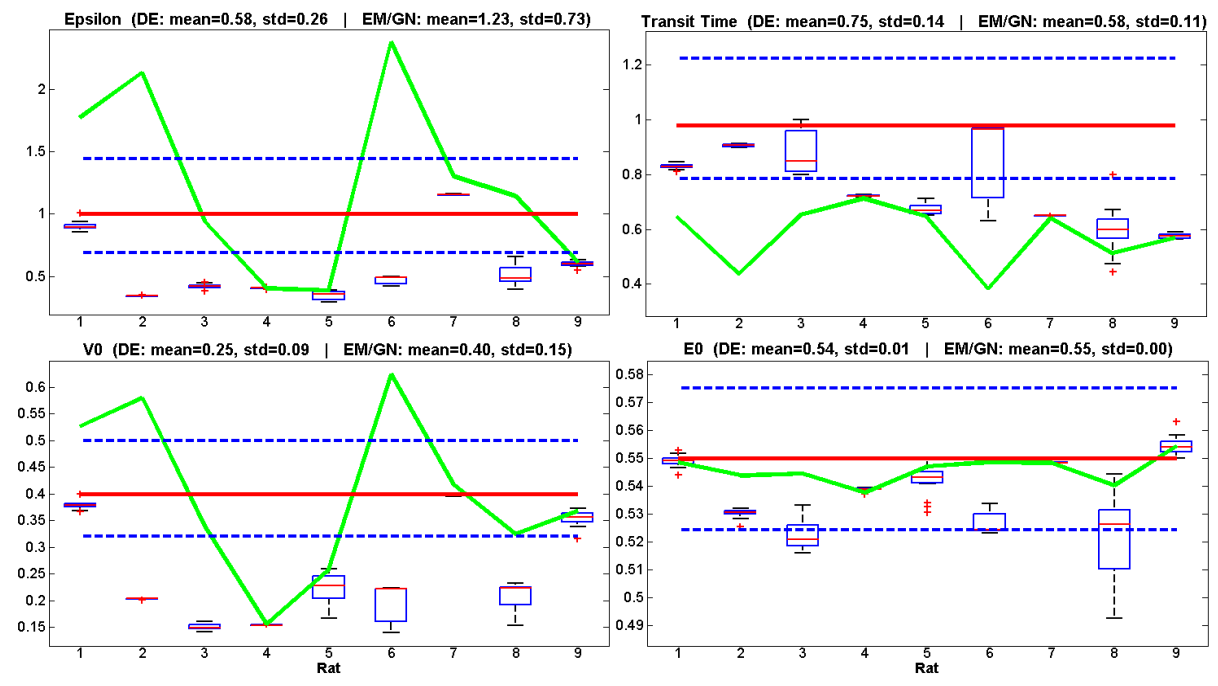

Fig. 1. Boxplots of the DE runs and EM/GN results (green line) for $\varepsilon$, transit time ( $t t$ ), $V_{0}$ and $E_{0}$. The parameter prior mean (resp. standard deviation) is indicated by the red (resp. blue) line.

venous (deoxygenated) fraction of that, such that a value of $2 \%$ actually seems much more realistic than the higher values of up to $6 \%$ estimated using EM/GN. Finally, both methods yield similar values for the resting oxygen extraction fraction. In summary, the estimates obtained from DE for these values seem both more realistic and more stable or at least as stable across sessions as the estimates from EM/GN. 


\section{Conclusion and future work}

A novel method to infer physiological parameters from observed BOLD signals has been described, showing the robustness and flexibility of global search optimization methods while being able to incorporate prior information in a principled Bayesian way. This has not been proposed before and could have a strong impact on a number of fMRI studies. Traditionally, these parameters are manually set or, only few of them, are determined by using conventional but potentially suboptimal local search methods like EM. Preliminary results on synthetic and real data showed promising results providing sensible and more stable parameter estimates. Possible future research includes testing on multimodal fMRI data, since information from cerebral blood flow and volume dynamics may help to further improve the reliability of the parameter estimates.

\section{References}

1. Buxton, R.B., Uludağ, K., Dubowitz, D.J., Liu, T.T.: Modeling the hemodynamic response to brain activation. Neuroimage 23, S220-S233 (2004)

2. Buxton, R.B., Wong, E.C., Frank, L.R.: Dynamics of blood flow and oxygenation changes during Brain activation: the balloon model. Magn Reson Med 39, 855-864 (1998)

3. Chumbley, J.R., Friston, K.J., Fearn, T., Kiebel, S.J.: A Metropolis-Hastings algorithm for dynamic causal models. Neuroimage 38(3), 478-487 (2007)

4. Coquery, N., Francois, O., Lemasson, B., Debacker, C., Farion, R., Rémy, C., Barbier, E.L.: Microvascular MRI and unsupervised clustering yields histology-resembling images in two rat models of glioma. J Cereb Blood Flow Metab 34(8), 1354-1362 (2014)

5. Das, S., Suganthan, P.: Differential Evolution: A Survey of the State-of-the-Art. IEEE T Evolut Comput 15, 4-31 (2011)

6. David, O., Guillemain, I., Saillet, S., Reyt, S., Deransart, C., Segebarth, C., Depaulis, A.: Identifying neural drivers with functional MRI: an electrophysiological validation. PLoS Biol 6(12), 2683-2697 (2008)

7. Eiben, A.E., Smith, J.E.: Introduction to Evolutionary Computing. Springer Verlag (2003)

8. Frau-Pascual, A., Ciuciu, P., Forbes, F.: Physiological models comparison for the analysis of ASL fMRI data. In: EEE International Symposium on Biomedical Imaging (ISBI) (2015)

9. Friston, K.J.: Bayesian estimation of dynamical systems: an application to fMRI. Neuroimage 16, 513-530 (2002)

10. Friston, K.J., Mechelli, A., Turner, R., Price, C.J.: Nonlinear responses in fMRI: the balloon model, Volterra kernels, and other hemodynamics. Neuroimage 12, 466-477 (Jun 2000)

11. Khalidov, I., Fadili, J., Lazeyras, F., Van De Ville, D., Unser, M.: Activelets: Wavelets for sparse representation of hemodynamic responses. Signal Process 91(12), 2810-2821 (2011)

12. Marreiros, A., Kiebel, S., Friston, K.: Dynamic causal modelling for fMRI: A two-state model. NeuroImage 39, 269-278 (2008)

13. Silvennoinen, M., Clingman, C., Golay, X., Kauppinen, R., van Zijl, P.: Comparison of the dependence of blood R2 and $R 2^{*}$ on oxygen saturation at 1.5 and 4.7 Tesla. Magn Reson Med 49(1), 47-60 (2003)

14. Stephan, K.E., Weiskopf, N., Drysdale, P.M., Robinson, P.A., Friston, K.J.: Comparing hemodynamic models with DCM. Neuroimage 38(3), 387-401 (2007)

15. Stephan, K., Kasper, L., Harrison, L., Daunizeau, J., den Ouden, H., Breakspear, M., Friston, K.: Nonlinear dynamic causal models for fMRI. NeuroImage 42(2), 649-662 (2008)

16. Vakorin, V.A., Krakovska, O.O., Borowsky, R., Sarty, G.E.: Inferring neural activity from BOLD signals through nonlinear optimization. Neuroimage 38(2), 248-260 (2007) 